\title{
Transport Properties of Vortices in Easy Flow Channels: A Frenkel-Kontorova Study
}

\author{
R. Besseling, R. Niggebrugge, and P. H. Kes \\ Kamerlingh Onnes Laboratorium, Leiden University, P.O. Box 9504, 2300 RA Leiden, The Netherlands
}

(Received 27 October 1998)

\begin{abstract}
Incommensurate easy flow channels in an otherwise perfect vortex lattice are investigated. The associated (point) defects in the lattice inside the channel cause an almost vanishing critical current, as shown by molecular dynamics simulations and a comparison with the Frenkel-Kontorova model. In addition to the normal flux flow behavior, we find a low mobility regime at small drives associated with defect motion. We treat this situation analytically for the case of a single defective vortex row. We also briefly discuss the relation to existing experiments on artificial vortex channels. [S0031-9007(99)08955-3]
\end{abstract}

PACS numbers: 74.60.Ge, 62.20.Fe, 74.60.Jg

In recent years, vast interest has grown in the dependence of static and dynamic properties of vortex matter on the spatial profile of the pinning potential originating from defects in the underlying material. The behavior of the vortex lattice was investigated experimentally as well as theoretically in the presence of a wide variety of these pinning landscapes, ranging from purely random [1-3] to highly periodic [4]. Several of these studies revealed the existence of static channels of easy vortex flow at currents just above the critical value $[2,3]$. In addition, this critical current was shown to be proportional to the interaction strength between vortices inside the channel and those in the channel edges (CE's), as expressed by the shear modulus of the vortex lattice $c_{66}[3,5]$. A precise theoretical description of this phenomenon and of its dependence on the channel width is still lacking. In this paper we study for the first time the properties of static easy flow channels within the framework of the Frenkel-Kontorova (FK) model [6]. It is demonstrated that a mismatch between channel width and lattice constants induces (point) defects in the channel leading to an almost vanishing shear strength. This can have important implications for the properties of vortex matter in a pinning potential with large spatial variations in strength. In addition, our results on the dynamics of incommensurate structures may be applied to various other fields, such as vortex dynamics in overdamped Josephson junction arrays, transport properties of charge density waves [7], and solid friction of confined layers [8,9].

We consider 2D vortices at $T=0$ in an easy flow channel of width $w$ and length $l$, confined by two semiinfinite pieces of a rigidly pinned vortex lattice with perfect hexagonal structure of lattice constant $a_{0}$ and row spacing $b_{0}=a_{0} \sqrt{3} / 2$. The pinned structures forming the edges are chosen to have their principal axis in the $x$ direction parallel to the channel and have a relative shift $\Delta x$ (left upper corner of Fig. 1a). First we describe qualitatively the case in which $w \approx b_{0}$ and $\Delta x=0$ (restricting the degrees of freedom for the mobile vortices to the $x$ direction), corresponding to the single chain FK model. Molecular dynamics (MD) simulations will then provide a justification of these qualitative arguments as well as show that our line of reasoning also applies to larger channel widths and a relative CE shift $\Delta x \neq 0$.

When a shear force is applied to the vortices in the channel, the onset of flux flow appears as the force exceeds the maximum shear stress. The maximum shear force density $F_{s}$ may be obtained from $c_{66}$ :
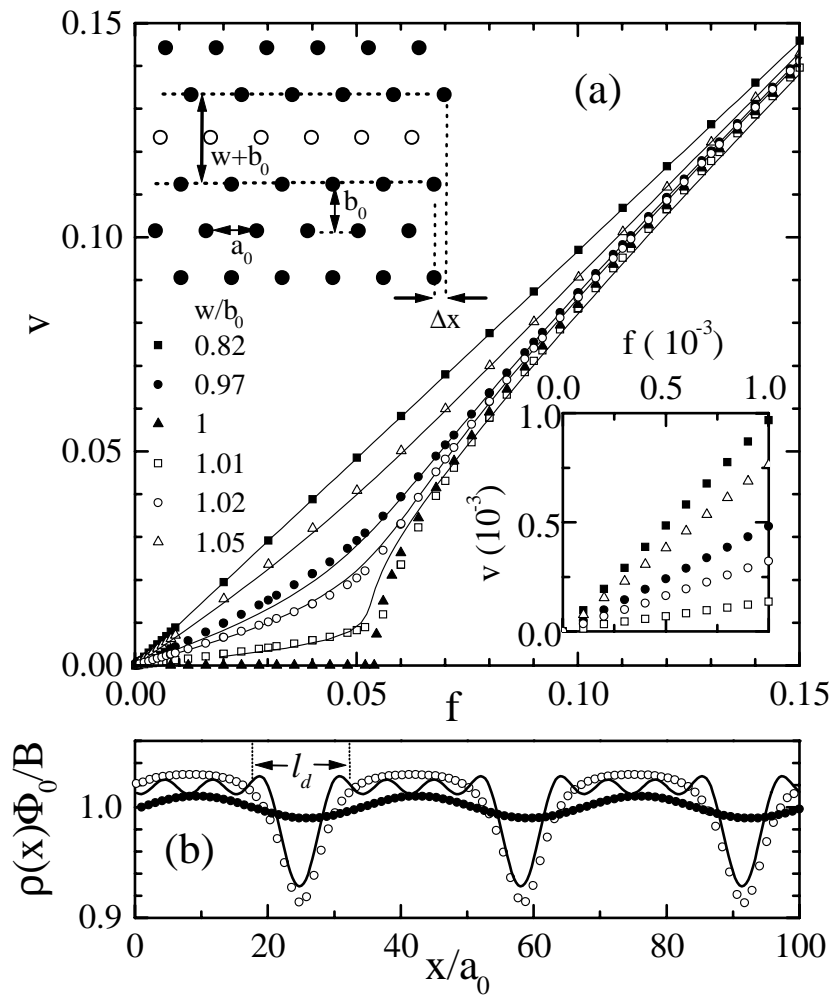

FIG. 1. (a) $f-v$ characteristics for vortex channels with $w \approx$ $b_{0}$ and $\Delta x=0$. Symbols are simulated data and drawn lines are obtained with Eq. (5). The inset shows an expanded view of the small velocity regime. Left upper corner shows the channel geometry with mobile vortices displayed by open symbols. (b) Vortex density $\rho(x)=1 /\left[w\left(x_{i+1}-x_{i}\right)\right]$ along the channel for $w=0.97 b_{0}$ : (o) GS as well as a snapshot of the moving row in the low mobility regime $(f=0.01) ;(\bullet)$ snapshot at large drive $(f=0.1)$. The drawn line represents $\rho(x)=$ $[w(h(\varphi+a)-h(\varphi)+a)]^{-1}$ at $v(f=0.01)$. 
$F_{s}=2 A c_{66} / w$ where $A$ accounts for the lattice anharmonicity and orientation of the shear direction with respect to the principal lattice axes [10]. For our orientation and a harmonic potential from the vortices in the CE's, it follows that $A=(\pi \sqrt{3})^{-1}$. Then, for $w \approx b_{0}$, the "edge" potential (per unit length) for a single vortex inside the channel can be defined as $U=\frac{\sigma}{2}\left[1+\cos \left(k_{0} x\right)\right]$, with $\sigma=a_{0}^{3} c_{66} /\left(\pi^{2} b_{0}\right)$ and $k_{0}=2 \pi / a_{0}$. Next, we consider the mutual interaction between the mobile vortices in the channel. These vortices have an average spacing $a$ which is given by the induction B as $a=\Phi_{0} /(B w)=a_{0} b_{0} / w$ with $\Phi_{0}$ the flux quantum. We use a harmonic approximation for the interaction, while its nonlocal nature is preserved by including interactions with $n$ neighbors in the chain. The effective spring constants $K_{n}$ then can be obtained from a London $1 / r$ interaction force between vortices: $K_{n}=\left(\Phi_{0}^{2} / 4 \pi \mu_{0} \lambda^{2}\right)(1 / n a)^{2}$ with $\lambda$ the penetration depth. Thus, in the presence of a transport current density $J$ applied perpendicular to the channel, the equation of motion for vortex $i$ in the channel reduces to an overdamped FK model,

$$
\gamma \dot{x_{i}}=f+\mu \sin \left(k_{0} x_{i}\right)+\sum_{n} K_{n}\left(x_{i+n}+x_{i-n}-2 x_{i}\right),
$$

where $f=J \Phi_{0}$ is the Lorentz force, $\mu=\pi \sigma / a_{0}$ is the amplitude of the periodic force, and the friction coefficient $\gamma$ is related to the flux flow resistance by $\gamma=B \Phi_{0} / \rho_{f f}(B)$.

In the FK model the critical force $f_{c}$ and the dynamic properties largely depend on both the value of $a / a_{0}$ as well as the dimensionless elastic constant $g=$ $a_{0}^{2} \sum_{n} n^{2} K_{n} / 2 \pi^{2} \sigma$ [7,11]. From the above given expressions for $\sigma$ and $K_{n}$ and using the low field expression for the shear modulus $c_{66}=\Phi_{0} B /\left(16 \pi \mu_{0} \lambda^{2}\right)$, we find that $g \approx(\lambda / a)\left(a_{0} / a\right)^{2}$ for our system. Since in practice [5] $\lambda / a_{0}>1$, we restrict ourselves to the case $g>1$ [12]. In case $a / a_{0}=1$, the vortex row locks in with the periodic potential and $f_{c}=\mu$. For rational $a / a_{0}$, the row contains point defects with a size $l_{d} \approx 2 \pi a \sqrt{g}$. Then $f_{c}$ is determined by their Peierls-Nabarro (PN) barrier [11] which, for an isolated defect and $g>1$ is given by $f_{c} \approx(8 / 3) \pi^{3} g \mu e^{-\pi^{2} \sqrt{g}}$. Accordingly, $f_{c} / \mu \ll 1$ and the channel has an almost vanishing critical current. Finally, for irrational $a / a_{0}$ and $n=1$, Aubry has shown that a transition exists to a state with $f_{c}=0$ as $g$ exceeds a critical value $g_{c}$. For $a / a_{0}=(\sqrt{5}-1) / 2, g_{c}=1$ [13]. The condition $g>1$ then implies that the vortex channel can have a truly vanishing critical current. The influence of nonlocal elastic constants [14] on the above results can be neglected as long as $l_{d} \gtrsim \lambda$, i.e., for $\lambda \lesssim 50 a_{0}$.

In order to check the above scenario, we performed MD simulations of easy flow channels in a thin film. The dynamics of a vortex inside the channel is governed by the following equation:

$$
\dot{\mathbf{r}}_{i}=f+\sum_{j \neq i} \mathbf{f}_{v}\left(\mathbf{r}_{i j}\right),
$$

with $a_{0}$ as unit of length, $\Phi_{0}^{2} /\left(2 \pi \mu_{0} \lambda^{2} a_{0}\right)$ as unit of force (per unit vortex length), and $4 \pi \mu_{0} \lambda^{2} /\left(\sqrt{3} \rho_{f f}\right)$ as unit of time. The external drive $f$ is directed along the channel. The interaction force is chosen as $f_{v}=$ $(1 / r)\left(1-r^{2} / r_{c}^{2}\right)^{2}$ with $r_{c}=3.33$ [1]. This interaction results in a shear modulus coinciding with the above given expression [in dimensionless units $c_{66}=1 /(4 \sqrt{3})$ ]. In Eq. (2), the index $j$ runs over all vortices, including those in the CE's (see Fig. 1a). We adopted periodic boundary conditions with $l$ large enough, such that our results did not depend on this length scale. For each $w$, we relaxed the system to its ground state (GS) and then measured the force-velocity $(f-v)$ characteristic by stepwise cycling the force $\left(f=0 \rightarrow f_{\max } \rightarrow 0\right)$ and taking the stationary velocity $v=\langle\dot{x}\rangle_{i, t}$ at each step.

Figure 1a shows the resulting $f$ - $v$ curves for $\Delta x=0$ and $w \approx b_{0}$. For $w=b_{0}$, the maximum shear strength is observed with a value $f_{c}=0.054$, coinciding with the dimensionless value of $\mu$ in (1). Additionally, the dynamics are identical to that of a single particle in a sinusoidal potential. Both observations are in agreement with the fact that the edge potential resulting from our vortex interaction is indeed harmonic. More interesting is the behavior in the incommensurate case $\left(w \neq b_{0}\right)$. As seen in the inset $f_{c} / \mu \lesssim 0.001$, which can be understood from our previous arguments when we calculate $g$ : expanding $f_{v}$ in (2) we obtain $K_{n}=\left(n a / a_{0}\right)^{-2}+0.18-0.003\left(n a / a_{0}\right)^{2}$, resulting in $g \approx 6$. A typical example of the GS defect structure is shown in Fig. 1b, where the open symbols represent the vortex density along the channel for $w=0.97 b_{0}$.

Turning to the dynamics in Fig. 1a, it is seen that the defective vortex rows possess a regime of low mobility in the $f-v$ curves. In this regime, transport in the row is carried by the defects which have a velocity $v_{d}$ much larger than the average velocity $v=c_{d} v_{d}$, with $c_{d}=\left|1-\left(a_{0} / a\right)\right|$ the defect density. A snapshot of the moving row then resembles the GS defect structure (see again the open symbols in Fig. 1b). A useful quantity here is the mobility of an isolated defect. From the simulation we get $v_{d} / f \approx 13$, a value which one can also obtain from the analytical treatment described below. The mobility of an interstitial is slightly larger than that of a vacancy due to the anharmonic vortex interactions. When entering the high mobility regime where $f \gtrsim \mu$, the defects are smeared out in a slight sinusoidal density modulation and the transport becomes coherent within the row (see the filled symbols in Fig. 1b).

It turns out that we can capture the transport characteristics within a perturbation theory as used in Ref. [15]. Consider Eq. (1). It is known that the motion of all particles in the FK chain can be completely described by the dynamic hull function $h(i a+v t)=x_{i}(t)-(i a+$ $v t)=h(\varphi)$, which represents the deviations of the particle positions from the undisturbed sliding values. From (1) we obtain the following equation for $h(\varphi)$ : 


$$
\begin{aligned}
\gamma v\left[1+h^{\prime}(\varphi)\right]=f & +\mu \sin \left\{k_{0}[\varphi+h(\varphi)]\right\} \\
& +\sum_{n} K_{n}[h(\varphi+n a) \\
& \quad+h(\varphi-n a)-2 h(\varphi)] .
\end{aligned}
$$

When mapping $\varphi$ back onto one period of the potential, $\tilde{\varphi} \equiv\left(\varphi-i a_{0}\right), h(\tilde{\varphi})$ has the periodicity $a_{0}$ resulting in

$$
f=\gamma v+\frac{\gamma v}{a_{0}} \int_{0}^{a_{0}}\left[h^{\prime}(\tilde{\varphi})\right]^{2} d \tilde{\varphi} .
$$

The shape and amplitude of $h(\tilde{\varphi})$ represent the defect structure of the moving FK chain and thus depend on $a / a_{0}, g$, and $v$. For large speed or in case of both large lattice mismatch and $g \gg 1$ (above Aubry's transition), $h(\tilde{\varphi})$ is sinusoidal with a small amplitude [9]. In our case, $h(\tilde{\varphi})$ can have a more complex shape as well as a large amplitude. We introduce higher orders in the perturbation to describe the dynamics for any $a / a_{0}$ by solving (3) with a trial hull function of the form $h(\tilde{\varphi})=\sum_{m=1}^{M}\left(H_{m} e^{i k_{0} m \tilde{\varphi}}+\right.$ c.c. $)$. Inserting this hull function in (3), we obtain a set of coupled equations for the coefficients $H_{m}$. Solving for $H_{m}$ yields the approximate form of $h(\tilde{\varphi})$. A full account of this work will be published elsewhere. Now the expression describing the $f-v$ characteristics is obtained by using this hull function in Eq. (4), which results for $M=3$ in

$$
f=\gamma v+\frac{2 \pi^{2} \mu^{2} \gamma v}{a_{0}^{2} \Omega_{c}^{4}+(2 \pi \gamma v)^{2}},
$$

where $a_{0} \Omega_{c}^{2}$ represents the elastic restoring force on nonlinear excitations in the FK chain. From the perturbation approach $\Omega_{c}$ is expressed in $\mu$ and the restoring forces on linear modes (phonons) at wave number $k=m k_{0}$,

$$
\Omega_{c}^{2}\left(a=a_{0} b_{0} / w\right)=\frac{\Omega_{1}^{2} \Omega_{2}^{2} \Omega_{3}^{2} a_{0}^{2}+\mu^{2} \pi^{2}\left(\Omega_{1}^{2}+\Omega_{3}^{2}\right)}{\Omega_{2}^{2} \Omega_{3}^{2} a_{0}^{2}+\mu^{2} \pi^{2}},
$$

in which $\Omega_{m}^{2}=\Omega^{2}\left(m k_{0}\right)=\sum_{n} 2 K_{n}\left[1-\cos \left(n m a k_{0}\right)\right]$. The last term in Eq. (5) corresponds to the sliding friction in the field of tribology [9]. It results from the dissipation of the internal (nonlinear) collective modes in the chain. The crossover from low to high mobility occurs at $v_{c}=$ $a_{0} \Omega_{c}^{2} /(2 \pi \gamma)$ where the amplitude of the hull function drastically decreases. In order to compare (5) with the simulated data, we use the previously obtained expression for $K_{n}$ and take into account that $\mu$ slightly depends on $w$ as well. The corresponding $f-\boldsymbol{v}$ curves, shown in Fig. 1a, agree well with the simulated data. The transport of a defective vortex row can thus be accurately described by perturbing the harmonic FK model.

In order to study channels of larger width, we adopted an edge shift $\Delta x(w)$ with a sawtooth shape $\left(0 \leq \Delta x \leq a_{0} / 2\right)$ which assures that, as we vary $w$, a perfect hexagonal structure is retained for $w=p b_{0}$ with $p$ an integer. However, for $w \neq p b_{0}$, the qualitative behavior did not depend on $\Delta x$. The inset of Fig. 2 shows the simulated $f$ - $v$ curves for $3.5 b_{0}<w<5 b_{0}$. The features are similar to the $f-v$ curves for $w \approx b_{0}$, and these are again associated with the presence of defects in the channel. Two characteristic structures can be identified: for $\left|\left(w / b_{0}\right)-p\right| \lesssim 0.4$ (I) the structure consists of $p$ vortex rows plus interstitials $\left(w / b_{0}>p\right)$ or vacancies $\left(w / b_{0}<p\right)$. The defects are not necessarily equally distributed within a row. In addition, the defect density differs per row due to the repulsive interaction with the channel edges. The latter is illustrated in Fig. 3a, where two snapshots of the motion for $w=$ $4.08 b_{0}$ in the low mobility regime are displayed with interstitials indicated by arrows. For $\left|\left(w / b_{0}\right)-(p+1 / 2)\right| \lesssim$ 0.1 (II), clustering of vacancies $\left(w / b_{0}>p+1 / 2\right)$ or interstitials $\left(w / b_{0}<p+1 / 2\right)$ occurs. As a result, alternating regimes form along the channel of $p$ and $p+1$ rows, which we refer to as stacking faults (SF's). The regimes are separated by dislocations with Burgers vectors at angles of $60^{\circ}$ with the channel direction, as shown in Fig. $3 \mathrm{~b}$.

Structure $I$ is just a $2 D$ extension of our results for a single incommensurate vortex row. The shear strength is almost vanishing due to the small PN barriers of the point defects in each row. The low mobility regimes in the $f-v$ curves once more originate from motion of these defects. However, two additional features appear. First, recalling our simple description of defect motion in a row $\left(v_{r}=c_{d, r} v_{d}\right)$, we see that, for equal defect velocities in different rows, a different defect density causes different average row velocities $v_{r}$, i.e., plastic motion within the channel. This clearly shows up in Fig. 3a, where the interstitials move at the same velocity $v_{d} \gg v_{r}$ while the row velocities vary from $v_{2}=0$ to $v_{3}=2 v$. Second, occasionally a hysteresis is observed in the transport curves

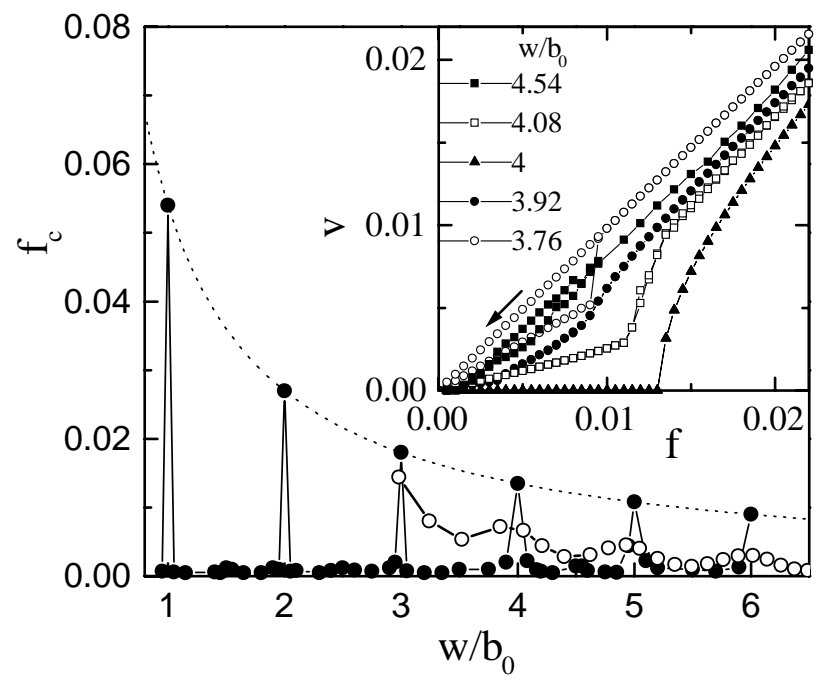

FIG. 2. Shear strength $f_{c}$ versus channel width for a velocity criterion of $5 \times 10^{-4}$ and $\Delta x=\Delta x(w)$. Open symbols are experimental data of artificial vortex channels at the same criterion [5]. The dashed line represents the continuum result: $f_{c}=\mu b_{0} / w$. The inset displays typical simulated $f-v$ characteristics. Note the hysteresis for $w=4.54 b_{0}$ and $w=3.76 b_{0}$. 


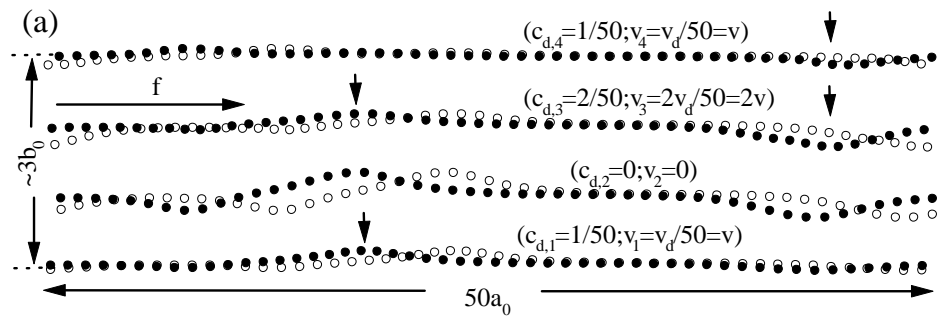

(b)

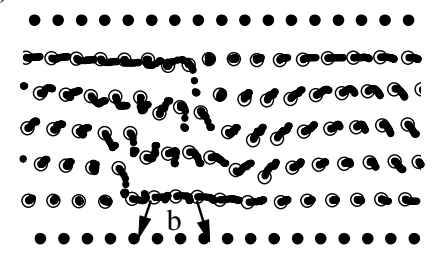

FIG. 3. (a) Two snapshots of the channel flow for $w=4.08 b_{0}$ (case I) in the low mobility regime $(f=0.007$, see inset of Fig. 2). For clarity the amplitude of vortex displacements from the average $y$ coordinate of a row has been enlarged by a factor of 3.5. Snapshot $2(\circ)$ is taken after an average displacement of $0.1 a_{0}$ with respect to snapshot $1(\bullet)$, whereas the defect displacement is about $6 a_{0}$. The rows are labeled with their interstitial density and average velocity. Defects are indicated by arrows. (b) ( $\circ$ ) defect structure at $w / b_{0}=4.54$ (case II), small dots represent the trajectories in the plastic regime at $f=0.002$.

of I (see inset of Fig. 2). This hysteresis originates from either a redistribution of defects between rows (for $0.2 \lesssim$ $\left.\left|w / b_{0}-p\right| \lesssim 0.4\right)$ or within the rows. After the transition, defects within the row have the maximum possible separation and they are clustered with those in neighboring rows, as expected from the standard FK model. Now the mobility of a defect moving along a locked (defect free) region of a neighboring row is small compared to that of a defect traveling along with a neighboring defect, since part of the periodic potential is destroyed in the latter case. This explains the transport hysteresis and the fact that the mobility is always larger after the transition. However, the nature of the transition and whether or not it occurs depend on both $\Delta x$ and $w$. In the high mobility regime of I, vortex transport is no longer carried by the defects but by the rows as a whole. They attain the same velocity and the flow loses its plastic character.

The SF's of II can locally form a commensurate structure. Then one naively expects $f_{c}$ to be of the same order as the commensurate value. The actual value, $f_{c} \approx \mu b_{0} /(15 w)$, is much lower because the SF's can move plastically by continuously creating and annihilating the dislocations at their borders. This is supported by the neighboring, defective rows: these rows "feed" the SF with point defects so that the longitudinal vortex displacements change into transverse displacements when entering the SF, as shown by the trajectories in Fig. $3 \mathrm{~b}$. Now the naive estimate, $f_{c} \approx \mu b_{0} /(2 w)$, marks the crossover to the regime where the SF's move coherently with the rest of the structure: $v_{\mathrm{SF}}=v$. The hysteresis, observed in the $f-v$ curve for $w=4.54 b_{0}$, is now caused by transitions in which the number of SF's decreases. For $f \downarrow$, this appears as a reduction in the amount of plastically moving SF's and thus an increase in mobility.

The main plot of Fig. 2 summarizes the behavior of $f_{c}$ vs $w$, where we took the maximum $f_{c}$ in case of hysteresis. The continuum result $f_{c}=\mu b_{0} / w$ applies only for $w=p b_{0}$ while $f_{c}$ of the incommensurate channels reflects our previous discussion of the barriers of the corresponding defects. To make a link to the experiments on samples with artificial vortex channels as used in Ref. [5], we also show the measured shear strength $f_{c}=2 \pi J_{c} \mu_{0} \lambda^{2} a_{0} / \Phi_{0}$ vs $w / b_{0}$ of such a device. It is seen that the commensurability peaks are lower and smeared out. Preliminary results of simulations in which positional disorder of vortices in the CE's is implemented indicate that pinning of defects (at $w \neq p b_{0}$ ) and generation of defects (at $w=p b_{0}$ ) by this (phase) disorder in the periodic potential forms the mechanism for the smoothening of $f_{c}$.

In conclusion, we have shown that the critical current of incommensurate vortex flow channels is drastically reduced as compared to the expected value for commensurate channels. The dynamic behavior exhibited a crossover from defect motion to coherent flow. We described these transport characteristics analytically for the case of a single vortex row.

We thank A. E. Koshelev, A. van Otterlo, and T. Droese for stimulating discussions.

[1] A. E. Koshelev and V.M. Vinokur, Phys. Rev. Lett. 73, 3580 (1994); S. Bhattacharya and M. J. Higgins, ibid. 70, 2617 (1993); M. Marchevsky et al., ibid. 78, 531 (1997); T. Giamarchi and P. Le Doussal, ibid. 76, 3408 (1996).

[2] H. J. Jensen et al., Phys. Rev. Lett. 60, 1676 (1988).

[3] H. J. Jensen et al., J. Low Temp. Phys. 74, 293 (1989).

[4] C. Reichhardt et al., Phys. Rev. Lett. 78, 2648 (1997).

[5] A. Pruyjmboom et al., Phys. Rev. Lett. 60, 1430 (1988); M. H. Theunissen et al., ibid. 77, 159 (1996).

[6] J. Frenkel and T. Kontorova, Zh. Eksp. Teor. Fiz. 8, 1340 (1938) [Phys. Z. Sov. 13, 1 (1938)].

[7] L. M. Floria and J. J. Mazo, Adv. Phys. 45, 505 (1996).

[8] O. M. Braun et al., Phys. Rev. B 56, 4987 (1997).

[9] T. Kawaguchi et al., Phys. Rev. B 56, 13932 (1997).

[10] J. Frenkel, Z. Phys. 37, 572 (1926).

[11] O. M. Braun et al., Phys. Rev. B 50, 13388 (1994).

[12] The case $g<1$ [11,13] describes the regime of large elastic instabilities [3], i.e., strong pinning of defects. This occurs for a vortex row in a strong, periodic potential [4].

[13] S. Aubry, Phys. D 7, 240 (1983); H. Peyrard and S. Aubry, J. Phys. C 16, 1593 (1983).

[14] E. H. Brandt, J. Low Temp. Phys. 28, 263 (1977); 28, 291 (1977).

[15] T. Strunz and F. Elmer, Phys. Rev. E 58, 1601 (1998). 